\title{
Properties of YBaCuO Nanobridges and dc-SQUIDs
}

\author{
Maxim V. Pedyash, Dave H.A. Blank, Jeroen H. de Muijnck, and Horst Rogalla \\ Department of Applied Physics, University of Twente, \\ P.O. Box 217, 5700 AE Enschede, the Netherlands.
}

\begin{abstract}
YBaCuO nanobridges and inductively shunted dc-SQUIDs have been patterned by direct Focused Ion Beam milling. Nanobridges manifest true Josephson effect, which can be explained by coherent motion of Abrikosov vortices in the bridge. An enhancement of this effect is proposed to be due to local suppression of the superconducting properties within the nanobridge area. This leads to a transition from SNS to SS'S type behavior of the nanobridge with decreasing temperature. Temperature dependence of the critical current, dynamic resistance, and SQUID voltage-flux modulation is discussed. The flux noise level of the nanoSQUID is measured to be about $6.5 \mu \Phi_{0} / \mathrm{VHz}$ at $62.7 \mathrm{~K}$.
\end{abstract}

\section{INTRODUCTION}

Nanobridges in high- $T_{c}$ superconducting thin films are very intriguing objects. They represent narrowings in the film with typical linear dimensions below $1 \mu \mathrm{m}$ which connect two superconducting banks. The nanobridge critical current density is equal to that of a bulk superconductor [1]. Electrical transport properties of nanobridges are determined by effects of the Abrikosov vortex motion, since lateral dimensions of the nanobridges are much higher than the superconducting coherence length $\xi$ [2]. At the same time, nanobridges manifest various aspects of the Josephson effect [1][3], which is normally attributed to weak links with significantly suppressed supercurrent.

It becomes commonly accepted that the coherent vortex motion in the nanobridge can be considered as a particular form of the Josephson phenomenon [4]. If motion of flux quanta in the nanobridge is strongly correlated, it causes the periodical time averaged current-phase relation.

In the presented article we discuss Josephson properties of the nanobridges. An oscillating dependence of the nanobridge critical current on applied magnetic field and microwave irradiation is considered as a direct proof of the Josephson coupling in these structures. SQUID performance of devices based on nanobridges is taken as an evidence of the Josephson effect as well.

\section{SAMPLE PREPARATION}

Nanobridges were structured in 50-100 nm thick (001)oriented YBaCuO films by direct Focused Ion Beam (FIB) milling. The nanobridge patterning procedure is discussed in details elsewhere [5]. The milled trench profile and dimensions of the nanobridges are determined using Scanning Force and Electron Microscopes (SFM and SEM). Typical

Manuscript received August 27, 1996 dimensions of the investigated nanobridges are: the length $1 \approx 150 \mathrm{~nm}$ and the width w down to $50 \mathrm{~nm}$ (Fig. 1(a)). Superconducting layers were sputtered by a pulsed laser deposition [6]. The transition temperature of as-deposited films is $(90 \pm 1) \mathrm{K}$.

Main disadvantage of the bridge patterning by FIB is strong implantation of beam ions into the film. This leads to suppression of superconducting properties of the nanobridge and degradation of the bridge in time.

Using nanobridges as weak links, SQUIDs were fabricated. An inductively shunted (directly coupled) single layer dc-SQUID geometry was chosen. The geometry of the nanoSQUIDs was optimized to reach the best operating performance. In Fig. 1(b) a photograph of the SQUID loop, made by FIB milling is presented.

\section{RESULTS AND DISCUSSION}

\section{A. Nanobridges}

Quadratic current-voltage (I-V) characteristics $V \propto\left(I-I_{c}\right)^{2}$ are typical for the nanobridges in the current range above the critical value $I_{c}$. The $I_{c}$ of the nanobridges was measured as a function of temperature. If reduced by $I_{c}$, measured at certain temperature, the critical current follows the same temperature dependence for nanobridges of different widths. $I_{c}(T)$ can be fitted by $I_{c} \propto\left(1-T / T_{c}\right)^{1.6 t 0.1}$ in a wide range of temperatures from $T_{c}$ down to at least $T_{c} / 2$. This observed universal temperature behavior coincides with data, obtained by other authors, and relates to the effect of flux creep in high- $T_{c}$ superconductors [7].

Temperature dependence of the nanobridge critical current is usually discussed in terms of the temperature dependence of the vortex edge barrier, since it is assumed that $I_{c}$ is the edge pinning critical current. $I_{c}$ is a function of the magnetic field penetration depth $\lambda$, which depends on temperature. In case of the hyperbolic nanobridges, an expression for $I_{c}$ is given by formula [8]:

$$
I_{c} \approx \frac{c^{2} \hbar}{8 \mathrm{e}} \cdot \frac{\mathrm{d}}{\lambda^{2}} \cdot \frac{1}{2} \sqrt{\mathrm{w} / \delta}
$$

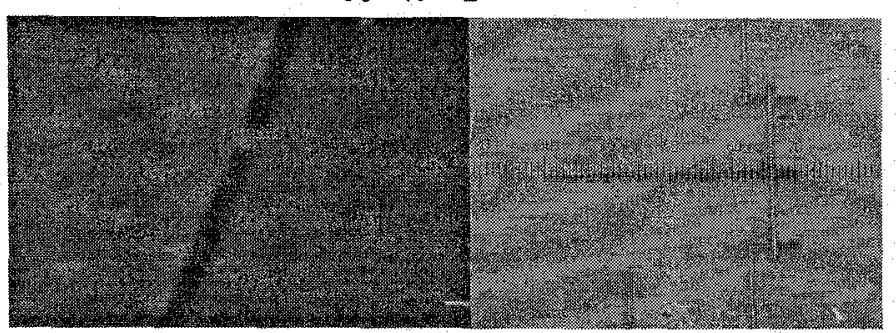

Fig. 1. Photographs of a nanobridge (left) and SQUID made by FIB (right). 


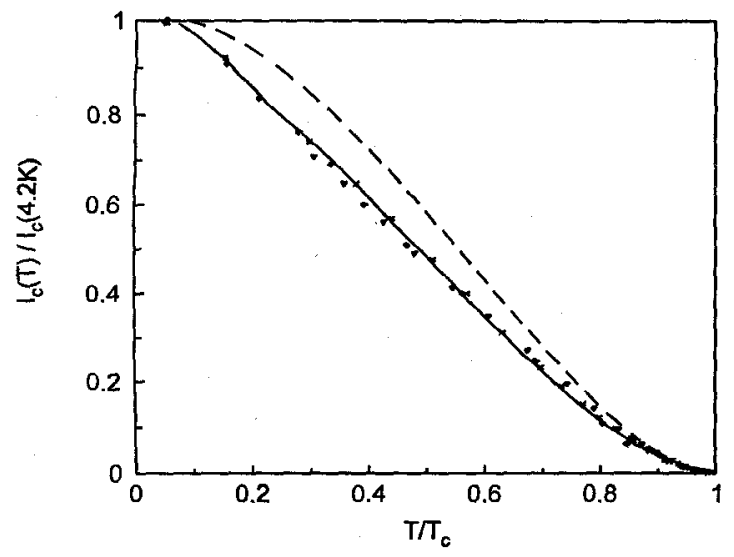

Fig. 2. Reduced critical current of three nanobridges as a function of reduced temperature. Solid line is calculated by taking thermal activation of vortices into account (see text).

Here, $d$ is the film thickness and $\delta$ is the nucleation distance of a vortex from the bridge boundary. An empirical approximation for $\lambda(\mathrm{T})$ was used in our model:

$$
\lambda(\mathrm{T})=\lambda_{0} \frac{1}{\sqrt{1-\left(\mathrm{T} / \mathrm{T}_{\mathrm{c}}\right)^{2}}} .
$$

From the temperature dependence of the period of the SQUID modulation the expression (2) was found to be valid in the whole temperature range $0<\mathrm{T}<\mathrm{T}_{\mathrm{c}}[5]$.

The results of calculation are plotted in Fig. 2 by a dashed line. According to (2), at lower temperatures $\lambda$ is almost temperature independent, and changes strongly only at $T$ close to $\mathrm{T}_{\mathrm{c}}$. This fact gives raise to a weak dependence of the calculated critical current on temperature at low temperatures, which contradicts the experimental data. It was found that $I_{c}$ significantly changes with temperature even at $\mathrm{T} \rightarrow 0$.

To explain the experimental results, a thermal assistance for vortices to overcome the edge energy barrier $U$ is assumed. At $I \approx I_{c}$, the $I-V$ characteristics of the nanobridge take form:

$$
\mathrm{V} \propto \mathrm{e}^{-\mathrm{U} / \mathrm{k}_{\mathrm{b}} \mathrm{T}},
$$

which is typical for a thermally activated process. The edge vortex pinning barrier $U$ was calculated from the vortex motion in the bridge [5]. Assuming thermal excitation of vortices in the nanobridge, the experimental temperature dependence of the critical current can be fitted well in the whole temperature interval $0<\mathrm{T}<\mathrm{T}_{\mathrm{c}}$ (a solid line in Fig. 2).

Small oscillation of the $I_{c}$ of the nanobridge was recently observed under influence of a weak magnetic field $\mathrm{H}$ (H $\leq 200$ Gauss). Data presented in Fig. 3 are obtained for a $300 \mathrm{~nm}$ wide bridge at $81 \mathrm{~K}$. The effective magnetic penetration depth $\lambda_{\text {eff }}(81 \mathrm{~K}$ ) is taken equal to $650 \mathrm{~nm}$ (for thin films $\lambda_{\text {eff }}=2 \lambda^{2} / \mathrm{d}$ ). Magnetic field $\mathrm{H}_{\Phi_{0}}$, which corresponds to a penetration of one flux quantum $\Phi_{0}$ into the nanobridge $\left(\mathrm{H}_{\Phi_{0}}=\Phi_{0} /\left(\mathrm{w} \cdot 2 \lambda_{\text {eff }}\right)\right)$, is estimated to be about 50 Gauss. This coincides with the observed period of the $I_{c}$ oscillation.

Shapiro-like steps appear in the I-V characteristics under microwave irradiation. Position of the steps is in an agree-

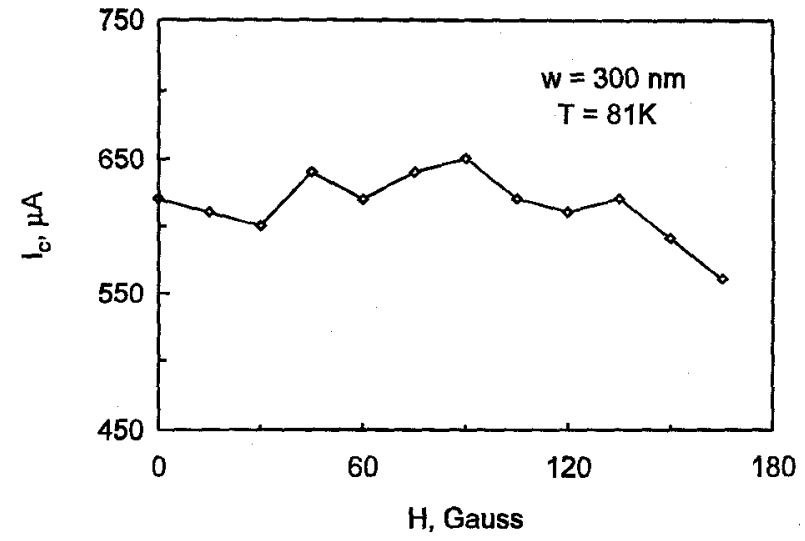

Fig. 3. Dependence of the bridge critical current on applied magnetic field.

ment with the Josephson voltage-frequency relation $V_{n}=n \Phi_{0} \cdot f(n=0,1,2 \ldots)$. Shapiro steps are observed up to the temperatures only $1 \mathrm{~K}$ below the critical temperature of the bridge. Up to 20 steps can be seen in the I-V characteristics. One should notice that $J_{c}$ of the nanobridges is a few orders of magnitude higher than that of any other types of known weak links (a typical $J_{c}$ of a nanobridge is about $(3 \pm 1) \cdot 10^{6} \mathrm{~A} / \mathrm{cm}^{2}$ at $\left.77 \mathrm{~K}\right)$.

Appearance of the steps in the I-V characteristics can be explained by synchronization of the motion of Abrikosov vortices by external microwave field. The model [9] predicts a linear dependence of the nanobridge critical current on the current $I_{r f}$ induced by $\mu$-wave irradiation, and non-oscillating dependence of the Shapiro steps amplitude on the irradiation power. A linear decrease of the critical current with an increase of the microwave-induced current is observed for our samples. However, the amplitude of $I_{c}$ as well as the amplitude of the first and second Shapiro steps reappears with further increase of the microwave power, after been suppressed to zero (Fig. 4). Such behavior directly follows from the periodical current-phase relation in the nanobridge, which means a presence of the Josephson component in the supercurrent.

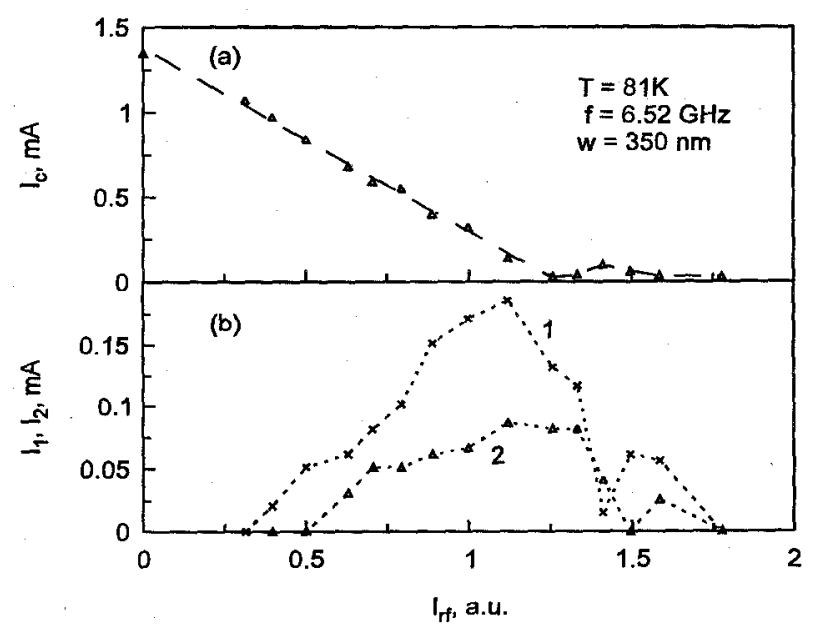

Fig. 4. Height of $I_{c}$ (a), $1^{\text {st }}$ and $2^{\text {nd }}$ Shapiro steps (b) as a function of the current induced by microwave irradiation. 


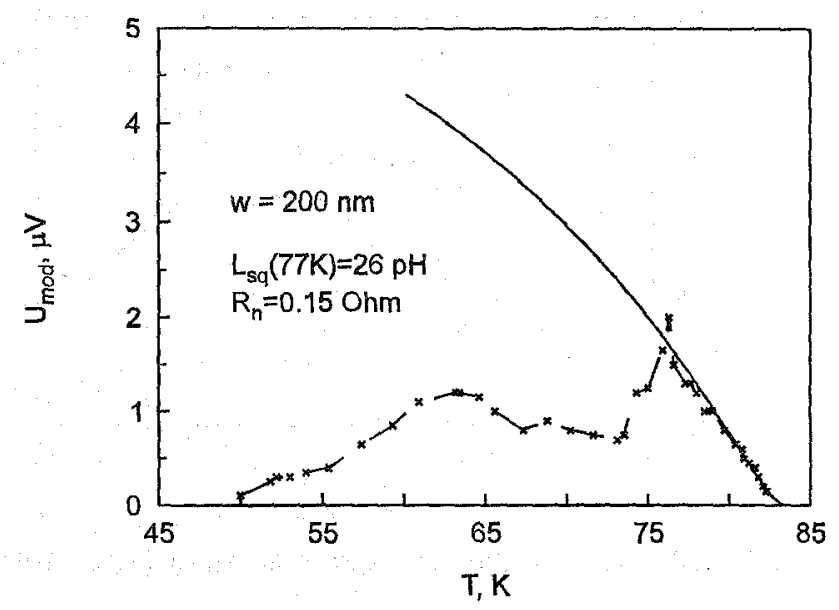

Fig. 5. Temperature dependence of $U_{\text {mod }}$ of a nanoSQUD. Solid line is calculated according to (4).

\section{B. NanoSQUIDs}

The I-V characteristics of nanoSQUIDs at zero external magnetic field are of the flux flow type, similar to those of single nanobridges. Devices with the nanobridge width $\mathrm{w}<300 \mathrm{~nm}$ manifest a SQUID voltage-flux modulation. The maximum measured $\mathrm{U}_{\text {mod }}$ was about $100 \mu \mathrm{V}(\mathrm{T}=40 \mathrm{~K})$.

An unusual dependence of the amplitude of the modulation on temperature $U_{\text {mod }}(T)$ was observed (Fig. 5). For wider bridges (just below $300 \mathrm{~nm}$ ), with decreasing temperature starting from $T_{c}$ of the bulk, the amplitude of the modulation increases rapidly. With further decrease of temperature the $\mathrm{U}_{\text {mod }}$ drops and vanishes, remaining zero at lower temperature. Narrower bridges show a maximum at higher temperature, a decrease of the voltage modulation in the intermediate temperature range and an abrupt raise at low temperatures (typically below $20 \mathrm{~K}$ ). This behavior is explained by suppression of superconducting properties in the nanobridge area. This leads to transition from SNS to SS'S type junction with decreasing temperature (see [1] for discussion). Systematic decrease of the nanobridge critical temperature and increase of the bridge resistivity with decreasing the nanobridge width confirms the degradation model.

The amplitude of the SQUID voltage modulation strongly depends on the dynamic resistance of the nanobridge. In contrast to conventional Josephson weak link SQUIDs, which are described by the RSJ model, nanoSQUIDs are typical flux-flow devices. Instead of a single well defined RSJ resistance parameter $R_{n}$, the nanobridge SQUID is characterized by its bias dependent dynamic resistance $R_{d y n}$. The $\mathrm{R}_{\mathrm{dyn}}$ is determined by effects of vortex flow in the bridge and can depend on temperature in a rather complicated way. It was found experimentally that $R_{\text {dyn }}$ raises strongly in, both, low and high temperature regions [1].

Some of the investigated SQUIDs showed an extremely narrow maximum in the temperature dependence of the $U_{\bmod }$ (Fig. 5). This maximum cannot be explained by a temperature variation of $\lambda$ or $\mathrm{I}_{c}$, which are found to be a gradual function of temperature. Probably, this sharp maximum can

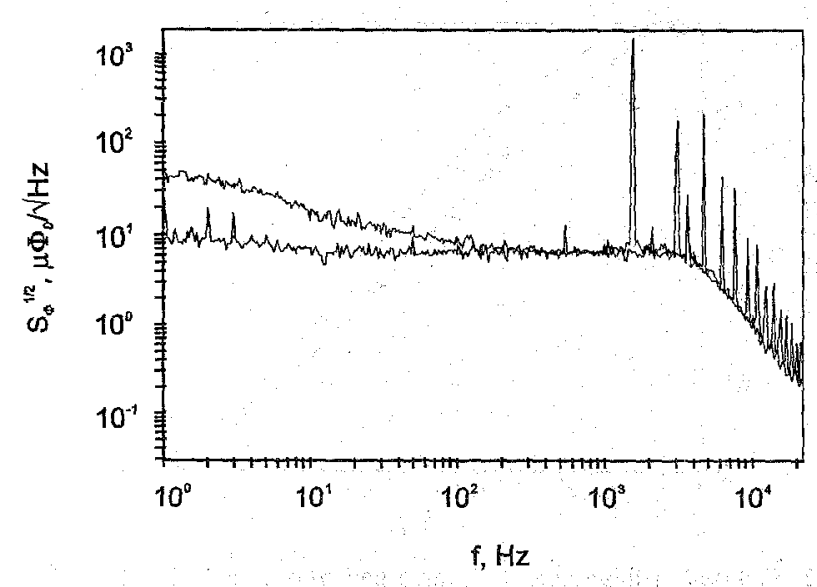

Fig. 6. Effective input flux noise spectrum of a nanoSQUID at $62.7 \mathrm{~K}$.

be explained by the liquid-glass transition in the vortex system with decreasing temperature [10]. This transition causes a sudden decrease of the resistance of the nanobridge, which might lead to an abrupt change of the $U_{\text {mod }}$.

Fit of the amplitude of the SQUID voltage modulation was made by the formula [11]:

$$
U_{\text {mod }}=\frac{7}{\pi^{2}} \cdot \frac{I_{c} R_{n}}{1+\beta_{L}}\left(1-3.57 \frac{\sqrt{k_{\mathrm{B}} T \cdot L_{s q}}}{\Phi_{0}}\right)
$$

The normal state resistance $R_{n}$ in (4) is substituted by the experimentally measured $R_{d y n}(T), L_{s q}$ is the SQUID inductance and $\beta_{\mathrm{L}}=2 \mathrm{I}_{\mathrm{c}} \mathrm{L}_{\mathrm{sq}} / \Phi_{0}$ is the SQUTD screening parameter. The $L_{\mathrm{sq}}$ is estimated to be about $20-30 \mathrm{pH}$ at $4.2 \mathrm{~K}$. It was found that to fit the experimental data only a small fraction (4-8\%) of the total nanobridge supercurrent should be of the Josephson origin, responsible for SQUID operation [5].

\section{SQUID Noise Characteristics}

Noise characteristics of nanoSQUIDs were measured using a flux locked loop technique [12]. In Fig. 6 an effective input flux noise spectrum, $\mathrm{S}_{\Phi}{ }^{1 / 2}(\omega)$ of a nanoSQUID at $62.7 \mathrm{~K}$ is given (the nanobridges width $\mathrm{w}=200 \mathrm{~nm}, \mathrm{~T}_{c}=85.3 \mathrm{~K}$ ). The amplitude of the $U_{\bmod }$ at this temperature is $4 \mu \mathrm{V}$. The white noise level is found to be about $6.5 \mu \Phi_{0} / \sqrt{H z}$. Applying a dcbias, the noise level at $1 \mathrm{~Hz}$ is about $35 \mu \Phi_{0} / \sqrt{H z}$. By using a bias reversal modulation scheme the noise level at $1 \mathrm{~Hz}$ can be reduced down to $8 \mu \Phi_{0} / \mathrm{VHz}$. This fact indicates that $1 / \mathrm{f}$ noise in our SQUIDs is caused by fluctuations of the junction critical current. A theoretical estimation of the white noise level using the following parameters: $(\partial V / \partial \phi)_{1}=10 \mu V / \phi_{0}$, $\mathrm{I}_{\mathrm{c}} / \mathrm{I}=2 / 3, \mathrm{~L}_{\mathrm{sq}}=26 \mathrm{pH}$, and $\mathrm{R}_{\mathrm{dyn}}=4 \mathrm{Ohm}$ gives the $\mathrm{S}_{\phi}{ }^{1 / 2}$ white noise of about $18 \mu \Phi_{0} / \sqrt{ } \mathrm{Hz}$, which is in the same order of magnitude as the measured noise level.

A source of the $1 / f$ noise, which might be relevant in case of the nanoSQUIDs, is the motion of vortices in the superconducting film, especially in the vicinity of the nanobridge. Vortices are weakly pinned and, if thermally activated, hop from one pinning site to another. A quasi-equilibrium system 
with variable relaxation time results in an overall $1 / \mathrm{f}$ power density spectrum of the noise [13].

Using values for an effective sensing area of the SQUID $A_{\text {eff }}$, derived from the period of the SQUID modulation $\left(A_{\text {eff }}(4.2 \mathrm{~K})=0.07 \mathrm{~mm}^{2}\right)[5]$, the magnetic field mean square root noise spectrum $S_{B}{ }^{1 / 2}(\omega)=S_{\Phi}{ }^{1 / 2}(\omega) / A_{\text {eff }}$ is calculated. The $\mathrm{S}_{\mathrm{B}}{ }^{1 / 2}$ is found to be $150 \mathrm{fT} / \sqrt{\mathrm{Hz}}$ at $1 \mathrm{kHz}$ and $184 \mathrm{fT} / \sqrt{\mathrm{Hz}}$ at $1 \mathrm{~Hz}$, being barely influenced by the $1 / \mathrm{f}$ contribution. Further optimization of the SQUID geometry can probably increase the field sensitivity of the devices.

\section{Josephson Effect in Nanobridges}

An oscillating dependence of the nanobridge critical current on the applied magnetic field and $\mu$-wave irradiation as well as the SQUID performance of nanobridge-based devices is a direct proof of the Josephson coupling in these structures.

There is an intrinsic difference in the origin of the Josephson behavior in structures with direct conductivity, depending on their dimensions compared to $\xi$. In narrow bridges $\left(w, 1 \approx \xi\right.$ ), if current higher than $I_{c}$ is applied, the entire nanobridge undergoes a transition to a normal state and exhibits a full spectrum of Josephson effects [14].

For wider bridges $(w, 1>\xi)$ the Josephson effect is caused by a periodic motion of flux quanta in the bridge. Ideally, this motion should be coherent due to a repulsive interaction between vortices [15]. Vortices, moving through the bridge as a rigid ensemble, cause the periodical dependence of the supercurrent on the time averaged number of vortices in the bridge. The commonly accepted criterion of the coherence in the vortex motion and, consequently, of the existence of the Josephson effect, is that the bridge lateral dimensions should be smaller than $\lambda_{\text {eff }}[16]$. However, the exact vortex interaction distance strongly depends on material properties, which determine the density of pinning sites and pinning strength. Thermal fluctuations affect coherent motion of vortices as well. In fact, the mentioned criterion is the upper limit for the observation of the coherent effects.

The investigated nanobridges (with the w of a few hundred nanometers) are the systems of an intermediate width. A "two fluid" model, where the Josephson component of the supercurrent coexists with a "strong" (i.e. non-periodic with the phase difference) term can be used for their description. A fraction of the Josephson current in the total supercurrent trough the bridge depends on temperature via the temperature dependence of $\lambda$. Suppression of superconducting properties of the nanobridge, leading to increase of $\lambda$, is known to enhance the Josephson effect in the nanobridges [1],[17].

\section{SUMMARY}

Nanobridges and dc nanoSQUIDs based on YBaCuO thin films were investigated. A periodical dependence of the nanobridge critical current on applied magnetic field and $\mu$ wave irradiation is considered as the direct proof of the Jo- sephson coupling in these structures. An enhancement of this effect can obtained by a local suppression of the superconducting properties within the nanobridge area.

Temperature dependence of the nanobridge critical current can be fitted by the model, assumed a thermal assistance for vortices to overcome the edge pinning barrier. Shapiro steps appear in the I-V characteristics under microwave irradiation and can be explained by synchronization of the Abrikosov vortices motion by external microwave field.

NanoSQUIDs showed well reproducible voltage-flux modulation, with the amplitude comparable to that of the devices, based of conventional weak links. The effective input flux noise of the nanoSQUID at $1 \mathrm{kHz}$ is about $6.5 \mu \Phi_{0} / \sqrt{\mathrm{Hz}}(\mathrm{T}=62.7 \mathrm{~K})$. This value is comparable with the best results, obtained for inductively shunted high- $T_{c}$ SQUIDs. Absence of significant $1 / \mathrm{f}$ branch in the noise spectrum makes nanoSQUIDs competitive to other devices at lower frequencies.

Main disadvantage of the nanobridge SQULD, patterned by the FIB process, is degradation of their superconducting properties with time. An average lifetime of these devices is approximately 1-2 months, if stored at room temperature. Preliminary results indicate that nanobridges, structured by electron beam lithography, are much more stable [5].

\section{REFERENCES}

[1] M.V. Pedyash, D.H.A. Blank and H. Rogalla, Appl. Phys. Lett., vol. 68 (8), pp.1156-1158, 1996.

[2] L.G. Aslamazov and A.I. Larkin, Sov. Phys.JETP, vol. 41, pp.381-396, 1975 [Zh. Eksp. Teor. Fiz., vol. 68, pp.766-775, 1975].

[3] J. Schneider, H. Kohlstedt and R. Wördenweber, Appl. Phys. Lett., vol. 63 (17), pp.2426-2428, 1993.

[4] A.N. Lykov, Advances in Physics, vol. 42 (3), pp.263-342, 1993.

[5] M.V. Pedyash, High- $T_{\mathcal{C}}$ superconducting nanobridges in $\mathrm{YBaCuO}$ thin films, Ph.D. thesis, University of Twente, Enschede, Netherlands, 1996.

[6] D.H.A. Blank, D.J. Adelerhof, J. Flokstra and H. Rogalla, Physica C, vol. 167, pp.423-432, 1990.

[7] A.A.R. Fernandes, I.N. Chan, J. Guimpel, O. Nakamura, D. Lederman, and I.K. Schuller, Appl. Phys. Lett, vol. 61 (26), pp.3181-3183, 1992.

[8] H. Rogalla, High $T_{C}$ Josephson contacts. Preparation and properties, Habilitation thesis, University of Giessen, Germany, 1986.

[9] A.T. Golovashkin and A.N. Lykov, Sov. Phys.-JETP, vol. 47, pp.110115, 1978 [Zh. Eksp. Teor. Fiz., vol. 74, p.214, 1978].

[10]D.S. Fisher, M.P.A. Fisher and D.A. Huse, Phys. Rev. B, vol. 43 (1), p.130, 1991.

[11]K. Enpuku, Y. Shimomura and T. Kisu, J. Appl. Phys., vol. 37 (11), pp.7929-7934, 1993.

[12] J. Clarke, Phys. B, vol. 126, pp.441-448, 1984.

[13]J. Clarke, SQUID concepts and systems, in Superconducting electronics, ed. H. Weinstoch and M. Nisenoff, Springer-Verlag, Berlin, Heidelberg, 1989.

[14]K.K. Likharev, Rev. Mod. Phys., vol. 51, pp.101-159, 1979.

[15]M.V. Pedyash, G.J. Gerritsma, D.H.A. Blank and H.Rogalla, IEEE Trans. Appl. Supercond., vol. 5 (2), pp.1387-1390, 1995.

[16]K.K. Likharev, Sov. Phys.-JETP, vol. 34, pp.906-912, 1972 [Zh. Eksp. Teor. Fiz., vol. 61, pp.1700-1711, 1971].

[17]A.J. Pauza, The fabrication of the Josephson junctions with an electron beam, Ph.D. thesis, University of Cambridge, Cambridge, United Kingdom, 1993. 